\title{
Implementation and Impact of Digital Pathology in the Pharmaceutical Industry
}

\author{
B.E. Maleeff
}

Department of Safety Assessment, GlaxoSmithKline, King of Prussia, PA

Digital pathology provides an alternative to traditional histopathology assessments, allowing users to view images of entire slide content, or selected fields at increasing magnifications, and to compare multiple slide images simultaneously. The technology incorporates robust image databases with metadata and search \& retrieval capabilities, and enables the use of digital slide images for cross-site collaboration and teleconsultation.

The Spectrum ${ }^{\mathrm{TM}}$ digital pathology information management platform (Spectrum; Aperio Technologies, Inc., Vista, CA) selected for use in GlaxoSmithKline R\&D Safety Assessment worldwide is an integrated system featuring digital slide scanning using the ScanScope XT digital slide scanner, Spectrum Plus ${ }^{\mathrm{TM}}$ slide image searchable database, ImageScope ${ }^{\mathrm{TM}}$ slide image viewer and Digital Slide Conferencing real-time collaboration tool. Implementation of Spectrum in the department took the efforts and cooperation of a team composed of members from the department, corporate Information Technology (IT) and the vendor. The system is designed to allow both internal and external collaboration, with appropriate firewall security controls.

Spectrum implementation occurred in 2 phases. Phase I implementation proceeded in a development environment, providing a platform for hardware and software testing, building metadata fields and pick lists, training of staff conducting data input and end users accessing digital slide data, development of documentation and troubleshooting. User access for Phase I was restricted to accounts within the corporate firewall. During the course of Phase I, feedback was collected from the development team and end users; this was used to optimize application performance and database design and improve user experience.

Phase II implementation consisted of installation of parallel hardware infrastructure and application software for the production environment. Additionally, corporate IT security controls were designed and put in place, ultimately allowing for secure application access for external (non-GSK) consultants. Based on learnings from Phase I, modifications and improvements were made to the user interface and metadata.

The direct impact of this digital pathology platform in a multi-site department is the ability for department colleagues to collaborate remotely in real time (Figure 1). This capability allows the company to minimize or eliminate (a) travel by pathologists for consultations and/or (b) shipping glass slides, impacting both shipping costs and the potential loss or breakage of slides in transit. Additional impact of Phase II implementation is enabling of real-time virtual collaboration with expert external consultants who have been issued secure network credentials.

By using digital pathology tools, timelines can be shortened appreciably, which in turn may impact the progression of a pharmaceutical compound in development either by permitting attrition at an earlier stage, or by allowing a promising compound to progress more rapidly to market. In either case, the financial impact to the business is measurable. 


\section{References}

[1] B.E.Maleeff and J.R.Ormand, Microsc.Microanal.16 (Suppl.2): 652-653, 2010.

[2] The author acknowledges the contributions of James R. Ormand and the support of Safety Assessment and IT management for the success of this project.

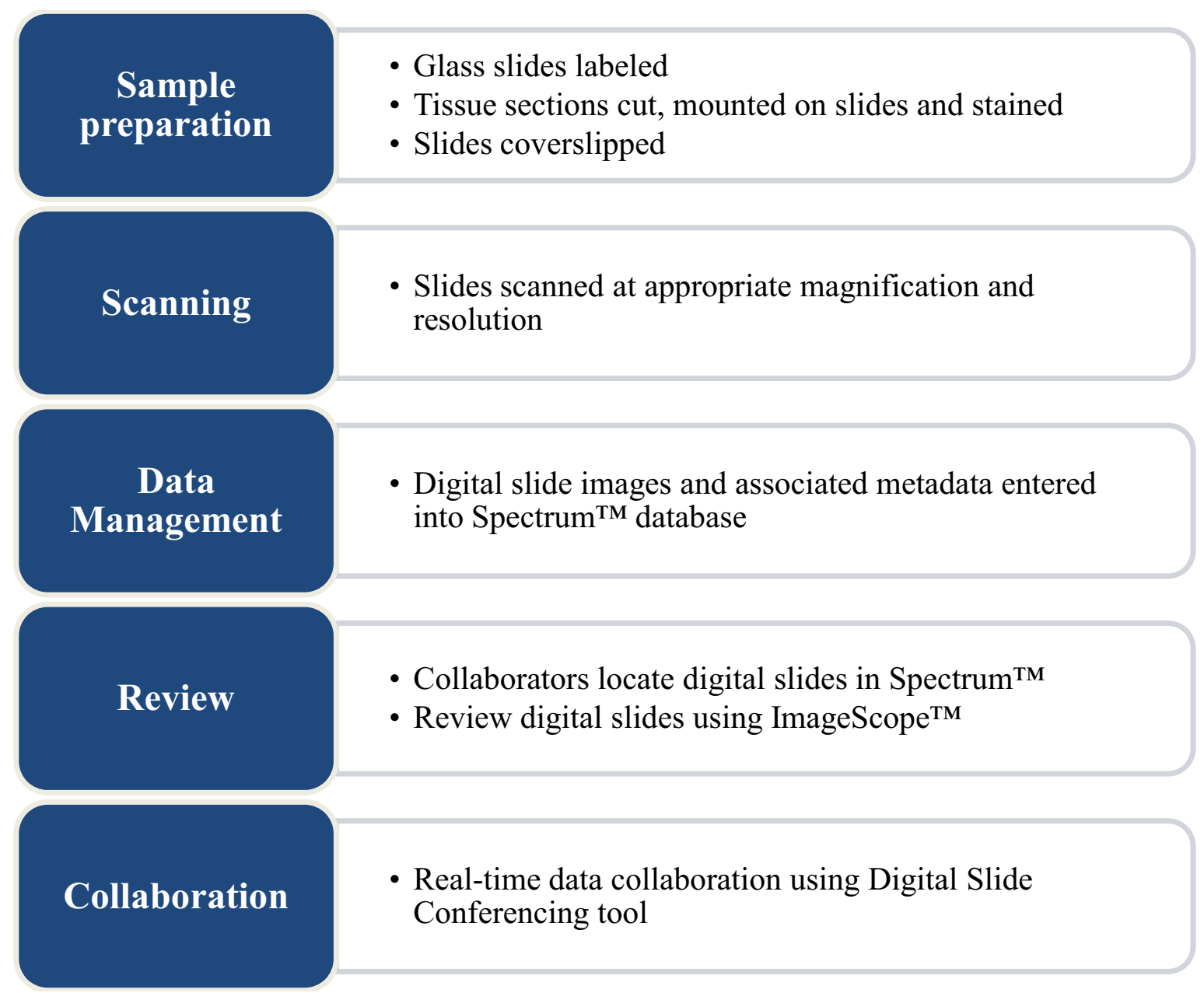

Figure 1. Digital pathology workflow schema. 